\title{
Linfoma suprarrenal primario bilateral: una presentación inusual
}

\author{
$M^{a}$. A. ALAMA ZARAGOZA, A. ROBLES INIESTA, I. ROCA ADELANTADO, $M^{\mathrm{a}}$ \\ A. SALES MAICAS*, $M^{\mathrm{a}}$. C. NAVARRO DE LEÓN, P. ROMÁN SÁNCHEZ \\ Servicios de Medicina Interna y *Anatomía Patológica. Hospital de Requena, SVS. \\ Requena. Valencia
}

\author{
BILATERAL PRIMARY ADRENAL LYMPHOMA: AN UNUSUAL PRE - \\ SENTATION
}

\section{RESUMEN}

Presentamos un caso de linfoma no Hodgking de localización exclusiva en ambas glándulas suprarrenales, con afectación de la reserva adrenal.

Esta inusual neoplasia, se asocia con una evolución rápida y fatal debido a múltiples complicaciones. En nuestro caso una hipercalcemia severa con hipotensión refractarias al tratamiento determinaron el óbito. Otras muchas complicaciones metabólicas que se presentan durante la evolución de esta neoplasia, podrían ser explicadas en el contexto del síndrome de lisis tumoral asociado al uso de elevadas dosis de corticoides ante clínica de insuficiencia adrenal.

Pese a su extremada rareza, esta entidad, debería incluirse en el diagnostico diferencial de las masas adrenales, unilaterales o bilaterales, ya que el diagnostico precoz es un factor clave para iniciar un tratamiento adecuado, evitar complicaciones potencialmente letales y tratar de aumentar la supervivencia de estos pacientes.

El empleo de técnicas de imagen y de la PAAF dirigida por ecografía o TAC, es actualmente la estrategia diagnóstica más válida aunque en la mayoría de los casos, el diagnostico definitivo se obtiene mediante el estudio necrópsico.

PALABRAS CLAVE: Linfoma no Hodgking. Glándulas Suprarrenales. Masas adrenales.

\begin{abstract}
A case of primary adrenal bilateral non-Hodgking lymphoma, with depressed adrenal reserve is pressented.

This rare neoplasm causes rapid evolution and fatal outcome in most cases.

In our patient, letal outcome was associated with severe hypercalce mia and refractary hypotension. Many other complications are due to tumoral lysis syndrome associated with high steroid doses in adrenal insufficiency.

This rare entity must be included in the differencial diagnosis of adrenal masses, uni or bilateral, because early diagnostic is important for preventing complications, potentialy lethals and for improving survi val. Image thecnics and ultrasound-guided or computed-tomographyguided FNA, are best diagnostic methods, but in many cases, deffinitive diagnostic is obtained by necropsy
\end{abstract}

KEY WORDS: Non-Hodgkin lymphoma. Adrenal glands. Adrenal mas ses.

Alama Zaragoza $M^{a}$ A, Robles Iniesta A, Roca Adelantado I, Sales Maicas $M^{a} A$, Navarro de León $M^{a}$ C, Román Sánchez P. Linfoma suprarre nal primario bilateral: una presentación inusual. An Med Interna (Madrid) 2002; 19: 524-526.

\section{INTRODUCCIÓN}

El linfoma primario bilateral de suprarrenales es extremadamente raro $(1-4,6,10,14,16)$, al contrario que la invasión secundaria por linfomas no Hodgkin, favorecida por la vascularización abundante de la glándula y la elevada concentración de esteroides. $(2,14)$. Esta inusual entidad, suele manifestarse como masas suprarrenales bilaterales grandes con o sin adenopatías $(8,4)$. Su sintomatología es muy inespecífica
$(1,5,9,11,14)$, cursando con deterioro del estado general, fiebre de origen desconocido, astenia, alteraciones gastrointestinales, hidroelectrolíticas y hematológicas (5), elevación de la LDH (12), del calcio sérico (13) y en ocasiones, clínica de insuficiencia suprarrenal.

El diagnóstico precoz es muy importante para que el tratamiento resulte efectivo, aunque su evolución suele ser muy poco favorable con una supervivencia media pese al tratamiento de 4-6 meses (13).

Trabajo aceptado: 3 de abril de 2001

Correspondencia: María Amparo Alama Zaragoza. C/ Juan Llorens, 12, puerta 28. 46008 Valencia. 


\section{CASO APORTADO}

Mujer de 73 años, que ingresó por deterioro del estado general. Presentaba desde hacía 8 meses, pérdida de peso, fiebre, astenia, anorexia y síntomas dispépticos. En los 2 últimos meses el cuadro asoció deterioro neurológico con desorientación temporo-espacial, tendencia a la agitación e hiponatremia con hipotensión. Como antecedentes personales refería: diabetes mellitus tipo 2 e hipertensión arterial bien controladas farmacológicamente.

En la exploración física destacaba importante deterioro del estado general, delgadez, desorientación, y $\mathrm{T}^{\mathrm{a}}$ ax. de $37,8^{\circ} \mathrm{C}$. Ausencia de adenopatías en las cadenas superficiales, signos de virilización o visceromegalias. Exploraciones complementarias: analítica: bioquímica sérica: $\mathrm{LDH}$ de $995 \mathrm{UI} / \mathrm{L}$ que llegó hasta $7.384 \mathrm{UI} / \mathrm{L}, \mathrm{Ca}^{+} 2$ sérico de 10,12 mg/dl que llegó hasta $23 \mathrm{mg} / \mathrm{dl}$, fósforo 4,31 mg/dl, ACTH de $589 \mathrm{pg} / \mathrm{dl}$ con cortisol de $18.9 \mathrm{mcg} / \mathrm{dl}$ y prueba de estimulación con ACTH negativa. $\mathrm{Na}+$ de hasta 125 con $\mathrm{K}+5,36 \mathrm{mmol} / \mathrm{l}$. Fosfatasa alcalina: $317 \mathrm{UI} / \mathrm{L}$. Beta 2 microblobulina 3169,6 ng/L. Determinación de Inmunoglobulinas: IgA 24 mg/dl, IgG 412 mg/dl, IgM 44 mg/dl. CAE, AFP y B-HCG negativas. Ac anti-tiroideos: negativos. Tiroglobulina y TSH normal. Transaminasas, parámetros de función renal, proteínas totales, albúmina, PTH, S-DHEA, aldosterona, hemocultivos, catecolaminas en orina, hemograma, hemostasia y gasometría venosa con bicarbonato y $\mathrm{PH}$ normales.

TAC cerebral: normal. IRM de área hipotálamo-hipófisis: normal. TAC torácico: normal. Gastroscopia y biopsia gástrica: normal. Ecografía abdominal: masas suprarrenales hipoecóicas, derecha de 84 x $34 \mathrm{~mm}$; izquierda de 60 x $38 \mathrm{~mm}$. TAC abdómino-pélvico: masas suprarrenales, derecha de 85 x $66 \mathrm{~mm}$; izquierda de 93 x $49 \mathrm{~mm}$ (Fig. 1). IRM suprarrenales: masas suprarrenales, derecha de $6 \times 4,8 \mathrm{~cm}$; izquierda de 5,7 x 4,5 cm. PAAF ecodirigida de suprarrenal izquierda: los preparados citológicos teñidos con Papanicolau y Giemsa mostraron celularidad abundante de tamaño variado y dispuesta individualmente. Se trataba de células con núcleos redondeados que mostraban frecuentemente nucleolo visible y citoplasma inaparente. Las células malignas se tipificaron inmunohistoquímicamente como de estirpe linfoide (positividad para CD45, con citoqueratinas AE1, AE3 y enolasa negativas). El estudio histológico tras biopsia con trucut de la suprarrenal derecha, confirmó el diagnóstico de proceso linfoproliferativo y permitió su tipificación. Se observó en las diferentes secciones histológicas, una infiltración difusa por elementos celulares atípicos de hábito linfoide. Junto a células linfoides grandes con prominentes nucleolos, se apreció una segunda población minoritaria de linfocitos pequeños (Fig. 2). Inmunofenotípicamente las células grandes fueron CD20 positivas y los linfocitos pequeños acompañantes fueron CD3 positivos. Un peque-

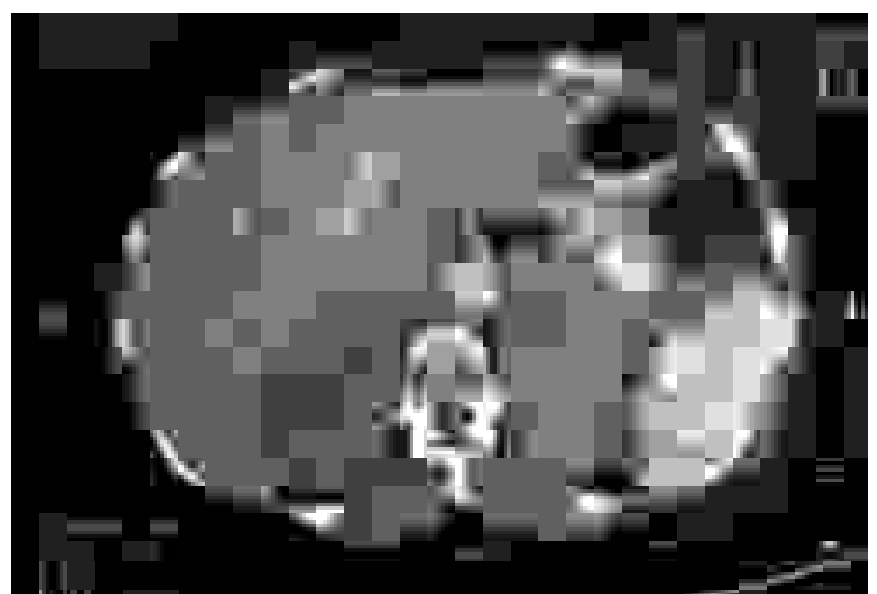

Fig. 1. TAC Abdominal realizado con contraste intravenoso en el que se aprecian masas suprarrenales bilaterales. La derecha de $85 \mathrm{x}$ $66 \mathrm{~mm}$ y la izquierda de $93 \times 49 \mathrm{~mm}$ discretamente inhomogéneas.

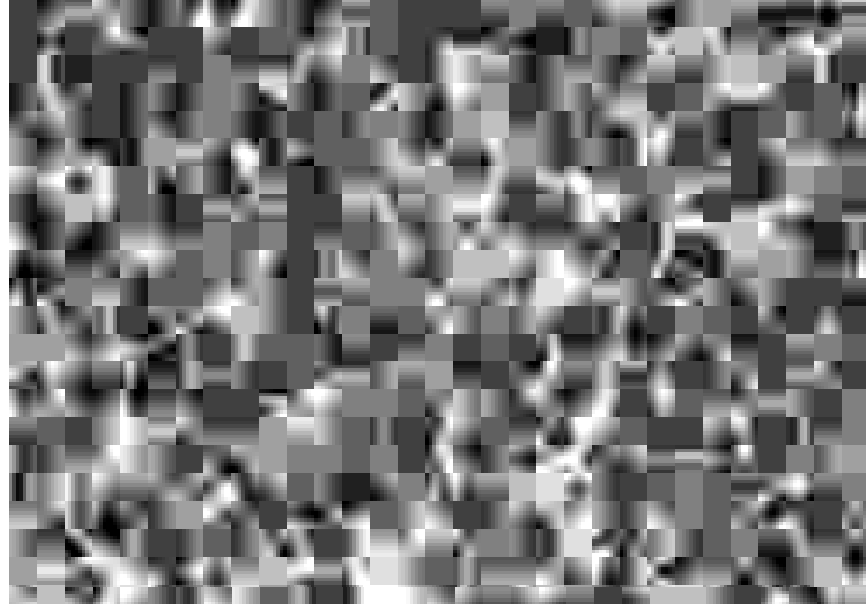

Fig. 2. Imagen histológica que presenta infiltración difusa por elementos linfoides en su mayor parte grandes, con núcleo redondeado y nucléolo prominente (Giemsa X 400).

ño porcentaje de células expresó bcl-2, así como la proteína p53 en aislados núcleos. Todo ello permitió tipificar la lesión como linfoma $\mathrm{B}$ difuso de célula grande de la tipificación REAL. Biopsia de médu la ósea: negativa para infiltración neoplásica.

Evolución: con el diagnóstico de linfoma primario suprarrenal bilateral mas Insuficiencia Suprarrenal, fue programada para adrenalectomía bilateral tras tratamiento sustitutivo, no pudiéndose realizar al presentar pocos días después cuadro de hipotensión e hipercalcemia refractarias al tratamiento que determinaron el fallecimiento.

\section{DISCUSIÓN}

El linfoma adrenal primario bilateral es prácticamente anecdótico (apenas unas decenas de casos publicados en la literatura mundial), aunque posible ya que estas glándulas poseen elementos heterotópicos linfoides (1). La edad de presentación se sitúa entre los 39-81 años, afectando por igual a ambos sexos (1). Debuta con clínica muy inespecífica como en nuestro caso, o como insuficiencia suprarrenal (8). Pese a la invasión de ambas suprarrenales, la sintomatología de déficit suprarrenal no es frecuente, ya que es necesaria la destrucción de al menos el $90 \%$ del tejido glandular. Es mucho más frecuente una disminución de la reserva adrenal, evidenciada en los estudios funcionales (1). El caso descrito por Shea en 1985 presentaba una baja reserva adrenal, puesta de manifiesto mediante respuesta inadecuada de cortisol a la estimulación con ACTH (18). El diagnóstico precoz es muy importante para evitar serias complicaciones, potencialmente letales. Muchas de estas complicaciones podrían explicarse dentro del contexto del síndrome de lisis tumoral, relacionado con el uso de altas dosis de corticoides (1) (en nuestro caso a la paciente se le llegaron a administrar hasta $300 \mathrm{mg} /$ día de hidrocortisona). Este síndrome, de evolución rápidamente progresiva, es más frecuente cuando el tumor es de gran tamaño, de crecimiento rápido y existe una $\mathrm{LDH}$ elevada. La presencia de hiperuricemia, acidosis láctica y fracaso renal, pueden ser explicadas en el contexto de este síndrome. Nuestra paciente presentaba un linfoma no Hodgkin de gran tamaño, con marcada elevación de la LDH, siendo éstos factores de riesgo para 
desarrollar lisis tumoral. Por ello, en general, el tratamiento sustitutivo hormonal debe realizarse con mucha precaución (1). El diagnóstico está actualmente basado en las técnicas de imagen, siendo en muchas ocasiones un hallazgo casual (inci dentaloma) (8). La ecografía, TAC, IRM $(6,9,17,19)$, así como la PAAF $(7,16)$ dirigida por ECO/TAC son las técnicas más válidas para el diagnóstico. Esta entidad debe estar incluida dentro del diagnostico diferencial de las masas adrenales uni o bilaterales $(1,11)$.

Previamente a la PAAF es necesario descartar un feocromocitoma por el peligro de desencadenar una crisis hipertensiva (1). El tipo histológico más frecuentemente descrito es el linfoma difuso de células grandes de la clasificación REAL, que en un $40 \%$ de los casos es de localización extranodal y típicamente presentan un crecimiento rápido de la masa tumoral $(1,20)$. Actualmente, gracias al avance en las técnicas de imagen, el diagnóstico de esta entidad se hace con más frecuencia premorten (10), aunque por desgracia, en la mayoría de las ocasiones, se sigue llegando al diagnóstico a través del estudio necrópsico.

La adrenalectomía bilateral más tratamiento quimioterápico coadyuvante, es el tratamiento de elección (3). La evolución de estos pacientes, es en general y pese al tratamiento, nefasta, teniendo una supervivencia media, desde el momento del diagnóstico, de 4-6 meses, existiendo tan solo un caso publicado en el que la paciente permaneció 52 meses en estado de remisión tras cirugía y quimioterapia (3).

Nuestra paciente falleció en 5 semanas tras el diagnóstico, con hipotensión e hipercalcemia severa de instauración brusca, que en este caso, descartadas radiológicamente las lesiones osteolíticas, se explicaría por la intervención de PTH-rP, IL-6, TNF y prostaglandinas, que aparecen durante la evolución del propio linfoma. Estas sustancias aumentan la actividad osteoclástica; además la insuficiencia suprarrenal, cursa con incremento del calcio sérico al aumentar la respuesta a la PTH de las células efectoras del túbulo renal, favoreciendo la reabsorción proximal del calcio por deplección de volumen. Se inició tratamiento hipocalcemiante intensivo y diálisis peritoneal pese a lo cual, la paciente sufrió una rápida evolución letal. En nuestro caso no disponemos de estudio necrópsico, pero la presencia de sendas masas suprarrenales, junto con la ausencia de infiltración neoplásica de médula ósea, y con estudios de imagen en los que no se objetivaron lesiones óseas, adenopatías ni visceromegalias, así como la patología y tipificación inmunohistoquímica de material de ambas suprarrenales, apoyan el diagnóstico de linfoma primario suprarrenal bilateral.

\section{Bibliografía}

1. López E, Piédrola G, González O; Palacios N, Cañizares A, López R. Linfoma suprarrenal primario: revisión a proposito de un caso. An Med Intern (Madrid) 1997; 14: 76-8.

2. Rodríguez Rodríguez JN, Fernandez Jurado A, Prados Madrona D. Linfoma suprarrenal primario. An Med Interna (Madrid) 1998; 15: 4229.

3. Wu HC, Shih LY, Chen TC, Chu SH, Tsai CC. A patient with bilateral primary adrenal lymphoma, presenting with fever of unknown origin and achieving long-term disease-free survival after resection and chemotherapy. Ann hematol, 1999; 78: 289-92.

4. Lee KS, Chung YS, Park KH, Kim HS, Kim HM. A case of primary bilateral adrenal lymphoma with partial adrenal insufficiency. Yonsei Med J 1999; 40: 297-300.

5. Baudard M, Pagnoux C, Audouin J, Buy JN, Bethoux JP, Delmer A Zittoun R. Idiopathic thrombocytopenic purpura as the presenting feature of a primary bilateral adrenal non Hodgkin's lymphoma. Leuk Lymphoma 1997; 26: 609-13.

6. Wang J, Sun NC; Renslo R; Chuang CC; Tabbarah HJ; Barajas L, French SW. Clinically silent primary adrenal lymphoma: a case report and review of the literature. Am J Hematol 1998; 58: 130-6.

7. Wu HH, Cramer HM, Kho J, Elsheikh TM. Fine needle aspiration cytology of benign adrenal cortical nodules. A comparison of cytologic findings with those of primary and metastatic adenal malignancies. Acta Cytol $1998 ; 42$ : 1352-8.

8. Maugendre D, Derrien C, Grulois I, et al. Primary adrenal lymphoma with latent adrenal insufficiency: a case report and literature review. Ann Endocrinol (Paris) 1998 ; 59: 34-9.

9. Hsu CW, Ho CL, Sheu WH, Harn HJ, Chao TY. Adrenal insufficiency caused by primary aggressive non-HodgKin's lymphoma of bilateral adrenal glands: report of a case and literature review. Ann Hematol
1999; 78: 151-4

10. Salvatore JR, Ross RS. Primary bilateral adrenal lymphoma. Leuk Lymphoma 1999; 34: 111-7.

11. Mizusawa H, Okaneya T, Yoneyama T, Taguchi I. Primary malignant lymphoma of the adrenal gland: a case report. Hinyokika Kiyo 1995; 41: 991-4.

12. Pimentel M, Johnston JB, Alland DR, Greenberg H Bernstein CN. Primary adrenal lymphoma associated with adrenal insufficiency: a distinct clinical entity. Leuk Lymphoma 1997; 24: 363-7.

13. Al Fiar FZ, Pantalony D, Shepherd F. Primary bilateral adrenal lymphoma. Leuk Lymphoma 1997; 27: 543-9.

14. Sanjuán F, Herrero A, Pérez A, Rubio A. Linfoma primario suprarrenal bilateral de presentación atipica como crisis Addisoniana. Med Clin (Barc) 1994; 105: 798 .

15. Dhote R, Tudoret L, Legmann P, Bachmeyer C, Christoforov B. Primary bilateral adrenal lymphoma as viewed by MRI. Apropos of a case J Radiol 1997; 78: 299-302.

16. Takai K, Hiragino T, Isoyama R, Takahashi M, Naito K. A case of primary adrenal lymphoma diagnosed percutaneous needle biopsy. Urol Int 1999; 62: 31-3

17. Kato H, Itami J, Shiina T, et al. MR imaging of primary adrenal lymphoma. Clin Imaging 1996; 20: 126-8.

18. Shea TC, Spark R, Kane B, Lange RF. Non-Hodgkin's lymphoma limited to the adrenal gland with adrenal insufficiency. Am J Med 1985; 78 : 711-4.

19. William F, Young Jr MD. Management appoaches to adrenal incidentalomas. Endocrinol Metabol Clin 2000; 29: 1.

20. Harris NL, Jaffe ES, Stein H, et al. A revised european-american classification of lymphoid neoplasm: a proposal from the international lymphoma study group. Blood 1994; 84: 1361-1392. 